\title{
Mercado potencial de serviços ancilares de potência reativa providos pelas usinas de açúcar e álcool
}

\author{
Marjhorre Laylakar Leyvosiyer Zinatto Malfatti*. Leonardo Ladeia Solera dos Santos**. Edmarcio Antonio Belati***. \\ Joel David Melo Trujillo*****.
}

\author{
* University Federal of ABC, Santo André, SP, 09210-580 \\ Brazil (Tel: 11-4472-1577; e-mail: jhorre3@, hotmail.com) \\ ** University Federal of ABC, Santo André, SP, 09210-580 \\ Brazil (Tel: 11-96451-9186; e-mail: leo.solera@hotmail.com) \\ *** University Federal of ABC, Santo André, SP, 09210-580 \\ Brazil (Tel: 11-4996-8244; e-mail: edmarcio.belati@ufabc.edu.br) \\ ****University Federal of ABC, Santo André, SP, 09210-580 \\ Brazil (Tel: 11-4996-0145; e-mail: joel.melo@ufabc.edu.br)
}

\begin{abstract}
The installation of sugar and ethanol plants has grown in recent years in Brazil because of incentives to new renewable sources that favor the supply of electric demand. These plants have several synchronous generators. In an adequate scenario of compensation for the injection reactive power for these generators, the injected reactive power can increase the reliability and safety of the electric system, improving the voltage profile of the bus near the sugar and alcohol plants. In order to assist in the analysis of the potential market for ancillary reactive power services, this paper presents a methodology that minimizes the amount paid by the electric power of the sugar and ethanol plants by adjusting the power factor of their generators and defining a compensation rate for the injected reactive power. This methodology uses two modules. The first is a spatial model to determine the plants closest to the substations that may need a greater amount of reactive power. The second is a particle swarm algorithm that finds the minimum value of the energy invoice payment for the plants found in the first stage. The methodology is applied in the São Paulo state, in order to find the compensation values for the plants of the regulated contracting environment that are closest to the $138 \mathrm{kV}$ substations. The result of the methodology is a map showing the plants that may have a greater contribution to improving the quality of the electric power supply and that will have a minimization in their energy bill. This class of results can help in the definition of a differentiated structure of remuneration per bus for the reactive power injection and in the analysis of the potential market of reactive power ancillary services provided by the sugar and alcohol plants.
\end{abstract}

Resumo: A instalação das usinas sucroalcooleiras tem crescido nos últimos anos no Brasil por causa dos incentivos a novas fontes renováveis que favoreçam o abastecimento da demanda elétrica. Tais usinas contam com diversos geradores síncronos. Em um cenário de remuneração adequada pela injeção de potência reativa para esses geradores, a potência reativa injetada pode aumentar a confiabilidade e segurança do sistema elétrico, melhorando o perfil de tensão das barras próximas a usina de açúcar e álcool. Com o intuito de auxiliar na analise do mercado potencial de serviços ancilares de potência reativa, neste trabalho se apresenta uma metodologia que minimiza o valor pago pela energia elétrica das usinas sucroalcooleiras por meio do ajuste do fator de potência de seus geradores e a definição de uma tarifa de remuneração pela potência reativa injetada. Tal metodologia utiliza dois módulos. O primeiro é um modelo espacial para determinar as usinas mais próximas das subestações que podem precisar de uma maior quantidade de reativos. Já, o segundo é um algoritmo de enxame de partículas que encontra o valor mínimo do pagamento da fatura de energia para as usinas encontradas na primeira etapa. A metodologia é aplicada no estado de São Paulo, a fim de encontrar os valores de remuneração para as usinas do ambiente de contratação regulada que estão mais próximas das subestações de $138 \mathrm{kV}$. O resultado da metodologia é um mapa que mostra as usinas que poderão ter uma maior contribuição na melhora da qualidade do fornecimento de energia elétrica e que terão uma minimização na sua fatura de energia. Esta classe de resultados pode ajudar na definição de uma estrutura diferenciada de remuneração por barra 
pela injeção de potência reativa e na análise do mercado potencial de serviços ancilares de potência reativa providos pelas usinas de açúcar e álcool.

Keywords: Biomass Plants, Transmission Lines, Geographic Information System, Electrical Substation, Structure of Compensation, Reactive Power, Ancillary Services

Palavras-chaves: Usinas de Biomassa, Linhas de Transmissão, Sistema de Informação Geográfica, Subestação de Energia Elétrica, Estrutura de Remuneração, Potência Reativa, Serviços Ancilares

\section{INTRODUÇÃO}

As usinas sucroenergéticas utilizam o bagaço da moagem e palha da cana-de-açúcar para produzir energia elétrica, injetando potência ativa na rede do sistema elétrico.

As Usinas de Biomassa possuem geradores síncronos instalados que também poderiam fornecer potência reativa contribuindo com o perfil de tensão das barras próximas à usina. $\mathrm{O}$ fornecimento não ocorre devido à falta de incentivos, poucas ações de políticas públicas e uma estrutura de remuneração que não viabiliza os investimentos dos usineiros (Hanashiro, D. Y.; Belati, E., 2018).

Os serviços ancilares auxiliam na operação eficiente do sistema elétrico, garantindo a estabilidade e a qualidade no fornecimento de energia elétrica (Brandão, R.; Oliveira, V. R.; Dorado, P.; Vardiero, P.; Carvalho, I., 2017). No Brasil, o Operador Nacional do Sistema Elétrico - ONS define esses serviços como: os controles primário e secundário de frequência, e suas reservas associadas; a reserva de prontidão; o suporte de reativo e auto-restabelecimento das unidades geradoras (ONS, 2009).

A ANEEL estabeleceu a tarifa de suporte de potência reativa para 2019 em R\$ 7,19/Mvar-h (ANEEL, 2018). Mas o valor proposto beneficia as grandes usinas e não desperta $\mathrm{o}$ interesse das pequenas usinas de geração distribuída. Tendo como referência o estudo, Metodologia para Remuneração do Serviço Ancilar de Suporte de Potência Reativa Quando Provido por Geradores, que informa que custo unitário anual de instalação de novas fontes de potência reativa é de 15,00 R\$ /MVAr (Hanashiro, D. Y.; Belati, E., 2018). Desta forma, analisar o mercado potencial de serviços de potência reativa providos por essa classe de usinas é a principal motivação deste trabalho.

O termo de mercado potencial é utilizado na área de marketing para quantificar os clientes potenciais que podem adquirir ou fornecer um determinado serviço (Kotler, 2000). Considerando a importância dos serviços ancilares de potência reativa para operação eficiente dos sistemas elétricos e o parque de usinas de sucroalcooleiras instaladas no Brasil, faz-se necessário uma análise do potencial do mercado da potência reativa que pode ser inserida pelos geradores dessa classe de usinas.

Neste trabalho propõe-se uma metodologia para análise mercado potencial de serviços ancilares de potência reativa providos pelas usinas de açúcar e álcool por meio de uma ferramenta de sistema de informação geográfica (SIG) e um algoritmo de otimização. Assim, a metodologia proposta consta de duas etapas. Na primeira etapa se identifica as usinas de biomassa próximas às subestações que precisem de reativos, considerando o zoneamento de cana-de-açúcar na região que influenciará no bagaço disponível para geração de energia elétrica. Já, na segunda etapa, se utiliza um algoritmo de enxame de partículas, PSO (siglas em inglês de Particle Swarm Optimization) para minimizar a fatura das usinas de açúcar e álcool encontradas na primeira etapa por meio do ajuste do fator de potência do gerador e propondo para os donos das usinas uma tarifa de remuneração pela potência reativa injetada. $\mathrm{O}$ resultado da proposta é um mapa com as usinas do ambiente de contratação regulada que poderão ter uma maior contribuição na melhora da qualidade do fornecimento de energia elétrica. Para garantir essa contribuição o planejador ou operador oferecerá para os donos da usina uma tarifa de remuneração que minimize sua fatura de energia. Esta classe de resultados proporciona aos planejadores do sistema da rede elétrica a definição de uma estrutura de remuneração para o setor sucroalcooleiro pela potência reativa que injetem no sistema elétrico, consequentemente, sendo possível analisar o mercado potencial desta classe de serviço ancilar.

\section{MÉTODOS PARA A AVALIAÇÃO DO MERCADO DE SEVIÇO ANCILAR DE SUPORTE DE REATIVOS}

Tendo como referência os trabalhos apresentados em: (Brandão, R.; Oliveira, V. R.; Dorado, P.; Vardiero, P.; Carvalho, I., 2017; Hanashiro, D. Y.; Belati, E., 2018; Khiaria, Besma; Jeguirimb, Mejdi, 2019; Cheng-Kang, Gao; Hong-Ming, Na; Kai-Hui, Song; Noel, Dyer; Fan, Tian, Qing-Jiang, Xu, 2019) é possível observar a importância do suporte de potência reativa nos sistemas elétricos e sua remuneração adequada para garantir a disponibilidade deste suporte.

Nos últimos anos, diversas metodologias têm sido propostas em ambientes SIG para auxiliar na estimação do potencial de novas fontes energéticas intermitentes (Cardoso de Lima, G.S.; Lopes, E. C.; Motta, J. G.; Asano Jr, R.; Valverde, M.; Suyama, R. Leite, P. T., 2018; Villavicencio, J.; Melo, J.; Padilha-Feltrin, 2018). A partir dessas estimações, os planejadores podem dar prioridade para os locais que podem ajudar no fornecimento da demanda elétrica. Para a análise do mercado potencial de serviço ancilares, a utilização dessa 
classe de ferramentas pode ajudar na identificação das fontes disponíveis para o fornecimento de potência reativa nos locais de maior demanda de potência reativa.

Este trabalho se diferencia dos trabalhos de valoração dos serviços ancilares, pois estabelece um modelo de avaliação espacial para encontrar as usinas cuja injeção de potência reativa terá maior impacto no sistema elétrico e diminuirá a fatura do consumo de energia elétrica, considerando que se tem uma remuneração diferenciada de acordo com sua localização, potência instalada e o nível de tensão no qual injete a potência. A metodologia proposta relaciona a necessidade de demanda reativa na zona de estudo, o preço de investimento para fornecer potência reativa e a energia elétrica consumida pela usina.

\section{METODOLOGIA PROPOSTA}

\subsection{Dados de Entrada}

Para implementação desta metodologia dois conjuntos de dados devem ser informadas. Por um lado, o conjunto de dados georreferenciados. Por outro, as informações relacionadas ao faturamento de energia elétrica das usinas.

No conjunto de dados referenciados, as informações são mapas ou camadas de informação com a localização georreferenciada e seus respectivos valores nominais de instalação para: as subestações elétricas com maior demanda de reativos e usinas térmicas de biomassa. Igualmente, forma parte deste conjunto o zoneamento agroambiental da cana-deaçúcar que regula a ocupação das terras na zona de estudo. Salienta-se que as camadas supracitadas devem estar na mesma projeção cartográfica, sendo possível a utilização de SIG para tal verificação e, caso seja necessário, realizar uma padronização de informações (Meehan, 2013).

Por outro lado, no conjunto de dados relacionados ao faturamento de energia devem ser informadas todas as tarifas por energia, demanda contratada e a energia consumida pela usina. Também, devem ser informados os limites máximos e mínimos para o fator de potência e a tarifa de remuneração pela injeção de potência reativa. Tais limites devem atender as recomendações técnicas e econômicas considerando a viabilidade da nova oportunidade para a remuneração de serviços ancilares.

\subsection{Etapa 1: Utilização do SIG para identificar Usinas mais próximas de Subestações com Demanda de Reativos}

O objetivo principal nesta etapa é identificar o conjunto de usinas de açúcar e álcool próximas das subestações que atendem uma maior quantidade de reativos no sistema elétricos. Os critérios para definir tais subestações e sua localização se encontram fora do escopo deste trabalho. Algumas recomendações relacionadas à análise de sensibilidade ou fluxo de potência ótimo podem ser explicadas em (Hanashiro, D. Y.; Belati, E., 2018). Igualmente, nesta etapa, considerou-se como critério que as usinas candidatas para atender as demandas de reativos são as usinas de maior potência instalada. Tendo em conta isto, a proposta calcula a distância destas usinas às subestações elétricas com maior demanda de reativos utilizando as ferramentas disponíveis em SIG, procurando encontra a mínima distância entre uma usina e uma subestação. $\mathrm{O}$ valor para tal distância é considerado como dado de entrada para esta etapa.

A maioria de SIG conta com ferramentas e filtros para atingir o objetivo desta etapa. Assim, as sequências de passos nesta etapa são:

Dados de entrada: Distância máxima a ser considerada entre uma usina e uma subestação; localização das subestações e usinas sucroalcooleiras.

Passo 1: Escolher a opção de cálculo de matriz de distância disponível no SIG a ser utilizado;

- $\quad$ Passo 2: Informar como ponto de origem as usinas e como destino ou alvo as subestações, definindo que se requer para cada usina a subestação mais próxima;

- Passo 3: Filtrar as usinas que sejam menores a distância máxima considerada.

Os passos supracitados podem ser implementados na maioria de SIG (Meehan, 2013). A saída desta etapa é uma camada de informação (mapa) com a localização geográfica da usina, sua potência instalada e distância a subestação. Tal camada é informada para a segunda etapa, a fim de realizar a minimização da fatura de energia, propondo uma nova opção de negócio para o dono de usina.

\subsection{Etapa 2: Minimização do faturamento de energia elétrica}

O objetivo principal desta etapa é minimizar a fatura de energia das usinas encontradas na Etapa 1 e que se encontram no ambiente de contratação regulada. Isto é, usinas que tem um contrato com a concessionaria de serviço de energia elétrica da região. Para atingir tal objetivo um algoritmo de PSO é utilizado.

Na formulação do problema de otimização, consideram-se as seguintes equações:

$$
\begin{array}{rl}
\operatorname{Min} f=T D x & P C \\
& +\sum_{h=1}^{24 \times 30}\left(T E_{1} x E C 1_{h}\right. \\
& -T E_{1} x E G 1_{h} \\
& +T E_{2} x E C 2_{h} \\
& -T E_{2} x E G 2_{h} \\
& \left.-T E R x\left(Q C_{h}-Q G_{h}\right) \times h\right)
\end{array}
$$

s.a.:

$$
\begin{array}{r}
T E R=\frac{T S A \times \frac{V_{\text {nom }}}{V_{\text {base }}} \times \frac{P_{\text {ins }}}{P_{\text {base }}}}{\frac{d_{\text {us }}}{d_{\text {base }}}} \\
Q G_{h}=\frac{E G T_{h}}{h} \times \tan (\operatorname{acos}(F P G))
\end{array}
$$




$$
\begin{gathered}
F P C_{h}=\cos \left(\operatorname{atan}\left(\frac{Q C_{h}-Q G_{h}}{\frac{E C T_{h}-E G T_{h}}{h}}\right)\right) \\
T E R_{\text {min }} \leq T S A \leq T E R_{\text {max }} \\
F P G_{\text {min }} \leq F P G \leq F P G_{\text {max }} \\
F P C_{\text {min }} \leq F P C_{h}
\end{gathered}
$$

\begin{tabular}{|c|c|}
\hline$T D$ & é a tarifa pela demanda ativa em $\mathrm{R} \$ / \mathrm{kW}$ \\
\hline$P C$ & é a demanda máxima registra no mês em $\mathrm{kW}$; \\
\hline$T E_{1}$ & $\begin{array}{l}\text { é a tarifa de energia no período de ponta em } \\
\mathrm{R} \$ / \mathrm{kWh} \text {; }\end{array}$ \\
\hline$E C 1_{h}$ & $\begin{array}{l}\text { é a energia ativa consumida em uma hora dentro do } \\
\text { período de ponta em } \mathrm{kWh} \text {; }\end{array}$ \\
\hline$E G 1_{h}$ & $\begin{array}{l}\text { é a energia ativa gerada em uma hora dentro do } \\
\text { período de ponta em } \mathrm{kWh} \text {; }\end{array}$ \\
\hline$T E_{2}$ & $\begin{array}{l}\text { é a tarifa de energia no período fora de ponta em } \\
\mathrm{R} \$ / \mathrm{kWh}\end{array}$ \\
\hline$E C 2_{h}$ & $\begin{array}{l}\text { é a energia ativa consumida em uma hora dentro do } \\
\text { período fora de ponta em } \mathrm{kWh} \text {; }\end{array}$ \\
\hline$E G 2_{h}$ & $\begin{array}{l}\text { é a energia ativa gerada em uma hora dentro do } \\
\text { período fora de ponta em } \mathrm{kWh} \text {; }\end{array}$ \\
\hline$T E R$ & $\begin{array}{l}\text { é a tarifa de remuneração pela energia reativa } \\
\text { injetada ao sistema elétrico em } R \$ \text { Mvarh; }\end{array}$ \\
\hline$Q G_{h}$ & $\begin{array}{l}\text { é a potência reativa injetada ao sistema elétrico em } \\
\text { Mvar; }\end{array}$ \\
\hline$Q C_{h}$ & $\begin{array}{l}\text { é a potência reativa consumida pela usina açúcar e } \\
\text { álcool em Mvar; }\end{array}$ \\
\hline$T S A$ & $\begin{array}{l}\text { Atual tarifa de remuneração pelos serviços } \\
\text { ancilares em R\$/Mvarh; }\end{array}$ \\
\hline$V_{\text {nom }}$ & $\begin{array}{l}\text { é a Tensão nominal da subestação elétrica mais } \\
\text { próxima à usina de açúcar e álcool e qual receberá } \\
\text { a potência reativa em } \mathrm{kV} \text {; }\end{array}$ \\
\hline$V_{\text {base }}$ & é a Tensão base em kV; \\
\hline$P_{\text {ins }}$ & $\begin{array}{l}\text { é a Potência instalada da usina de açúcar e álcool } \\
\text { em kW; }\end{array}$ \\
\hline$P_{\text {base }}$ & é a Potência base em kW; \\
\hline$d_{u s}$ & $\begin{array}{l}\text { é a Distância entre a subestação elétrica mais } \\
\text { próxima à usina de açúcar e álcool qual receberá a } \\
\text { potência reativa em km; }\end{array}$ \\
\hline$d_{\text {base }}$ & é a Distância base em km; \\
\hline
\end{tabular}

\begin{tabular}{|c|c|}
\hline$F P G$ & $\begin{array}{l}\text { é a Fator de potência do gerador síncrono instalado } \\
\text { na usina de açúcar e álcool; }\end{array}$ \\
\hline$E G T_{h}$ & $\begin{array}{l}\text { é a Energia ativa total gerada em uma hora em } \\
\text { kWh; }\end{array}$ \\
\hline$E C T_{h}$ & $\begin{array}{l}\text { é a Energia ativa total consumida em uma hora em } \\
\text { kWh; }\end{array}$ \\
\hline$F P C_{h}$ & $\begin{array}{l}\text { é o Fator de potência resultante da usina de açúcar } \\
\text { e álcool medido desde no ponto de conexão com a } \\
\text { rede elétrica; }\end{array}$ \\
\hline$T E R_{\min }$ & $\begin{array}{l}\text { é o valor mínimo para a tarifa de remuneração de } \\
\text { energia reativa injetada ao sistema elétrico em } \\
\text { R\$/Mvarh; }\end{array}$ \\
\hline$T E R_{\max }$ & $\begin{array}{l}\text { é o valor máximo para a tarifa de remuneração de } \\
\text { energia reativa injetada ao sistema elétrico em } \\
\mathrm{R} \$ \text { Mvarh; }\end{array}$ \\
\hline$F P G_{\min }$ & $\begin{array}{l}\text { é o valor mínimo para o Fator de potência do } \\
\text { gerador síncrono instalado na usina de açúcar e } \\
\text { álcool; }\end{array}$ \\
\hline$F P G_{\max }$ & $\begin{array}{l}\text { é o valor máximo para o Fator de potência do } \\
\text { gerador síncrono instalado na usina de açúcar e } \\
\text { álcool; }\end{array}$ \\
\hline$F P C_{\min }$ & $\begin{array}{l}\text { é o valor mínimo para o Fator de potência } \\
\text { resultante da usina de açúcar e álcool visto desde } \\
\text { da concessionaria de energia para cada hora. }\end{array}$ \\
\hline
\end{tabular}

Sendo que:
A formulação supracitada minimiza a fatura do mês de energia da usina, considerando que o dono da usina recebe um valor de remuneração $T E R$ pela injeção de potência reativa na rede. Assim, considera-se nesta formulação que se tem a curva de carga para cada dia do mês como os valores de energia ativa e reativa consumida e gerada em cada hora. Igualmente, as tarifas $T D, T E_{1}$ e $T E_{2}$ são parâmetros de entrada definidos no contrato de compra de energia elétrica.

O valor de $T E R$ é calculado pela Equação (2), considerando a potência instalada da usina $P_{\text {ins }}$, sua distância com respeito a subestação $d_{u s}$ e a tensão da subestação $V_{\text {nom }}$ na qual impactará os reativos injetados pela usina. Em geral, as usinas estão conectadas nos níveis de tensão de demais instalações de transmissão (DIT). Uma das características da DIT é ser extensa e radial até chegar à subestação do sistema de transmissão. Assim, o valor de $V_{\text {nom }}$ é aquele correspondente à linha de transmissão que conecta a DIT com a usina de açúcar e álcool.

Observe-se que a partir (2), usinas mais próximas, com maior capacidade e em níveis de tensão altos receberão uma maior remuneração. Os valores bases de $V_{\text {base }}, P_{\text {base }}$ e $d_{\text {base são }}$ utilizados para que essas grandezas sejam convertidas em por unidade (adimensional). Dessa forma, as unidades de TER dependerá dos valores de $T S A$ que é a tarifa de remuneração 
pelos serviços ancilares. No Brasil, a cada ano se define um valor para tal tarifa (ANEEL, 2018). Desta forma, neste trabalho se considera que o valor de $T E R$ será proporcional a $T S A$, dentro de uma faixa definida por (5).

A Equação (3) permite obter o valor da potência reativa para cada hora $Q G_{h}$ a partir do valor de fator de potência do gerador $F P G$. Assim, dependendo do valor de $F P G$, uma quantidade de potência reativa poderá ser gerada. Tal fator se encontra dentro de uma faixa de variação definida pela Equação (6). A variação de (6) depende de restrições técnicas e de recomendações por parte dos operadores e engenheiros da usina. A definição desses limites não faz parte do escopo deste trabalho, algumas recomendações podem ser encontradas em (Hanashiro, D.; Belati, 2018).

Com o intuito de não incrementar as perdas elétricas no sistema elétrico as concessionárias limitam o fator de potência visto desde o ponto de conexão para um valor mínimo. Caso o consumidor se encontre abaixo desse valor, então se teria que pagar uma tarifa de remuneração pelo excedente de potência reativa (ANEEL, 2010). Desta forma, na formulação se considera que o valor de $F P G$ deve ser ajustado de forma que o fator de carga medido pela concessionária $F P C_{h}$ seja maior a um valor mínimo, como se observa em (7).

A partir de (1) até (7) o valor mínimo será encontrado a partir de valores apropriados de FPG e TSA, sendo conhecidas como variáveis de decisão (Silva de Souza, Jeane; Molina, Yuri Percy; Silva de Araujo, Clivaldo). Para encontrar esses valores um algoritmo de PSO foi utilizando. O PSO se fundamenta em que cada partícula pode mudar sua posição de acordo ao conhecimento dos vizinhos que tenham visitado as melhores posições locais (Silva de Souza, Jeane; Molina, Yuri Percy; Silva de Araujo, Clivaldo; Pereira de Farias, Wendell; Santos de Araujo, Luri 2018). Neste trabalho, uma quantidade de partículas é alocada na faixa de variação de FPG e TSA. Desta forma, em cada iteração do algoritmo se muda a posição das partículas dentro das faixas definidas por (5) e (6). Cada partícula é atraída à melhor posição $g^{*}$ para minimizar o valor de (1), cumprindo com as restrições (2) até (7). Igualmente, Em cada iteração do PSO, a partícula na posição $x_{i}^{(t)}$ se movimenta a uma nova posição $x_{i}^{(t+1)}$ por meio da seguinte equação:

$$
x_{i}^{(t+1)}=x_{i}^{(t)}+v_{i}^{(t+1)}
$$

Na qual, $v_{i}^{\left({ }^{(+1)}\right.}$ é a nova velocidade de cada partícula para iteração $(t+1)$, sendo determinada por:

$v_{i}^{(t+1)}=w \cdot v_{i}^{(t)}+c_{1} \cdot r_{1} \cdot\left[g^{*}-x_{i}^{t}\right]+c_{2} \cdot r_{2} \cdot\left[x_{i}^{*(t)}-x_{i}^{t}\right]$

Em que: $w$ é o fator de ponderação, conhecido como peso de inercia porque caracteriza a tendência do movimento das partículas; $v_{i}{ }^{(t)}$ é a velocidade na iteração $t$, sendo chamada como velocidade de partida; $c_{1}$ e $c_{2}$ são os coeficientes de aceleração que permitem a cada partícula em uma interação $t$ sair de uma região com solução local do problema e ir à solução global, $r_{1}$ e $r_{2}$, são dois números aleatórios dentro de um intervalo de 0 a 1 .

As equações (8) e (9) procuram encontrar a melhor solução global entre todas as posições percorridas pelas partículas. Desta forma, o problema modelado pelas Equações (1) até (7) é resolvido pelo PSO seguindo o algoritmo ilustrado na Fig. 1. O critério de parada desse algoritmo é número máximo de iterações. Sugestões para calibração desse número são explicadas em (Jeane; Molina; de Araujo; de Farias; de Araujo, 2018). Ao final da execução de tais iterações, o valor mínimo de (1) é encontrado.

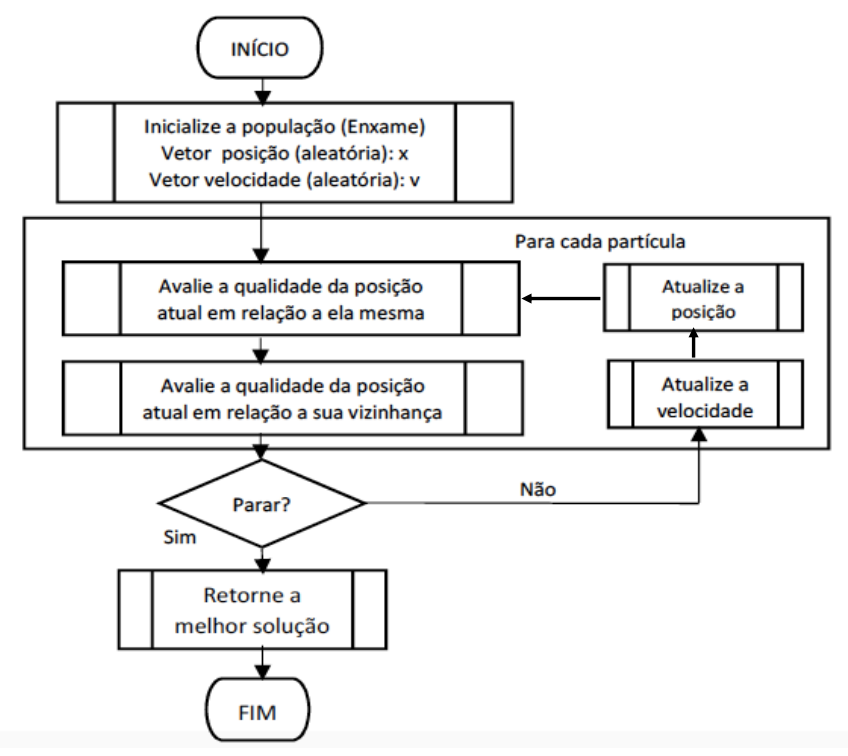

Fig. 1 Algoritmo do Enxame de partículas (PSO).

\section{APLICAÇÃO DA PROPOSTA}

A metodologia proposta foi aplicada no estado de São Paulo para analisar o mercado potencial de serviços ancilares de potência reativa providos pelas usinas de açúcar e álcool dentro de um raio de $10 \mathrm{~km}$ das subestações de $138 \mathrm{kV}$. A Etapa 1 da metodologia proposta foi executa no software livre QGIS versão 3.6.2, considerando as camadas de informação disponíveis no sites da Empresa de Pesquisa Energética, EPE (EPE, 2009), portal GeoSeade da Fundação Sistema Estadual de Análise de Dados, SEADE, (GEOSEADE, 2019) e no site da União da Indústria de Cana.-deAçúcar (UNICA, 2016) A saída dessa etapa é mostrada na Fig. 2 e Tabela 1.

A escala de cores da Fig. 2 foi escolhida para representar o zoneamento agroambiental disponível em (Secretaria de Infraestrutura e Meio Ambiente do Estado de São Paulo, 2019). Observa-se que as usinas encontradas pela proposta se encontram dentro de zonas adequadas e adequado com limitações, círculos de cor amarela nessa figura. Isto se deve a que foi inserido no software QGIS um filtro para considerar tais zonas, a fim de considerar usinas com disponibilidade de cana para o fornecimento de potência reativa. Atualmente, 
caso uma usina não forneça o valor contrato de potência reativa seria penalizado por meio de uma multa. Assim, casos de indisponibilidade foram excluídos desta análise do mercado potencial.

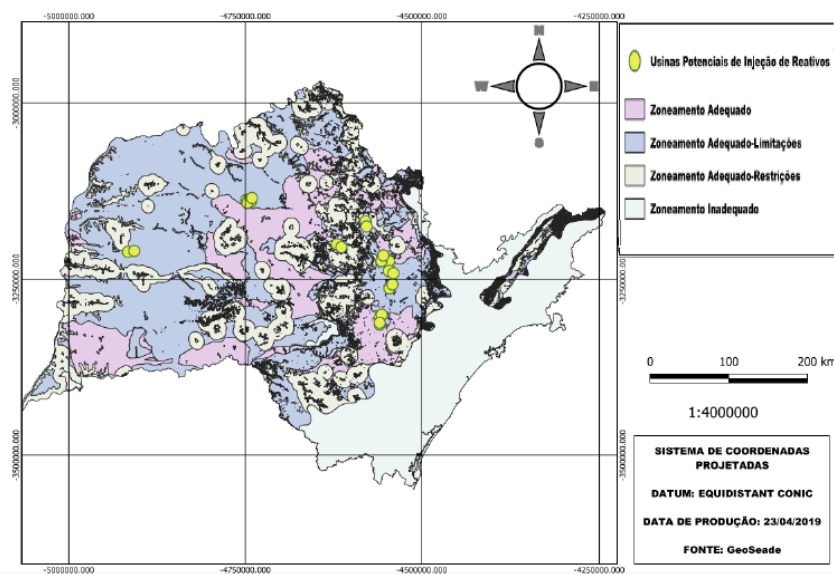

Fig. 2 Subestações candidatas à receber injeção de potência reativa das usinas de Biomassa.

$\mathrm{Na}$ Tabela 1, mostram-se todas as usinas que se encontram dentro do raio de $10 \mathrm{~km}$ de cada usina com linhas de $138 \mathrm{kV}$. Por questões de privacidade de informação, um apelido é utilizado para cada usina.

\begin{tabular}{|c|c|c|c|}
\hline $\begin{array}{l}\text { Nome da } \\
\text { Usina }\end{array}$ & $\begin{array}{l}\text { Nome da } \\
\text { Subestação }\end{array}$ & $\begin{array}{c}\text { Distancia } \\
(\mathrm{km})\end{array}$ & $\begin{array}{c}\text { Potência } \\
\text { Instalada da } \\
\text { Usina (MW) }\end{array}$ \\
\hline Usina 1 & Tiete & 4,21 & 2,40 \\
\hline Usina 2 & Val Paraiso & 8,53 & 4,20 \\
\hline Usina 3 & Cerquilho III & 2,47 & 3,80 \\
\hline Usina 4 & Porto Ferreira & 7,41 & 2,18 \\
\hline Usina 5 & $\begin{array}{c}\text { Santa Barbara } \\
\text { D'Oeste }\end{array}$ & 6,56 & 3,60 \\
\hline Usina 6 & Limeira & 2,33 & 5,00 \\
\hline Usina 7 & Araras & 8,13 & 4,97 \\
\hline
\end{tabular}

Tabela 1: Resultado da aplicação de Matriz Distância Usinas e Subestações do QGIS.

Para cada uma das 7 usinas mostradas na Tabela 1 foi executado o algoritmo da Fig. 1. Na Fig. 3, ilustra-se o perfil de carga por unidade (P.U.) para todos os dias do período de safra das usinas consideradas na aplicação da proposta. Tal perfil é um valor médio dos perfis de demanda das usinas da Tabela 1.

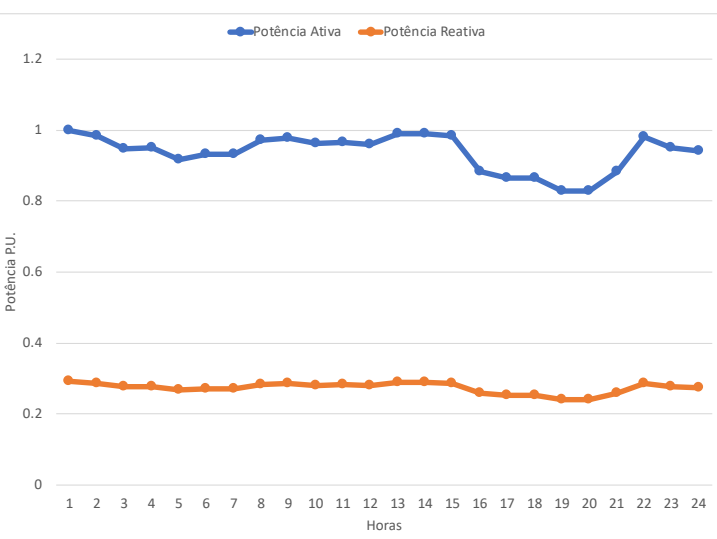

Fig. 3 Potência ativa e reativa demanda por cada usina sucroalcooleiras analisada.

As tarifas consideradas e parâmetros do PSO são mostrados na Tabela 2. Considerou-se que as usinas pertencem ao grupo de consumidores de classe A. Por outro lado os parâmetros do PSO foram determinados após diversas iterações. Cada vez que se executa o algoritmo da Fig. 2 uma nova resposta se obterá para a fatura de energia. Desta forma, o algoritmo foi executa uma 100 vezes para encontrar o valor com maior frequência que se repete nessas execuções.

\begin{tabular}{|c|c|}
\hline Parâmetro & Valor \\
\hline$T D$ & $13,80593 \mathrm{R} \$ / \mathrm{kW}$ \\
\hline$T E_{1}$ & 0,50867 R\$/kWh; \\
\hline$T E_{2}$ & 0,3074 R\$/kWh; \\
\hline$T S A$ & 7,19 R\$/Mvarh; \\
\hline$V_{\text {base }}$ & $138 \mathrm{kV}$ \\
\hline$P_{\text {base }}$ & $100 \mathrm{~kW}$ \\
\hline$d_{\text {base }}$ & 10 km; \\
\hline$T E R_{\min }$ & 7,19 R\$/Mvarh; \\
\hline$T E R_{\max }$ & 9,50 R\$/Mvarh; \\
\hline$F P G_{\min }$ & 0,9 \\
\hline$F P G_{\max }$ & 1 \\
\hline$F P C_{\min }$ & 0,92 \\
\hline$c_{1}$ & 1 \\
\hline$c_{2}$ & 1,5 \\
\hline$w$ & 0,02x(número aleatório); \\
\hline $\mathrm{N}$ & 200 iterações; \\
\hline M & 25 partículas; \\
\hline
\end{tabular}

Tabela 2: Informações de entrada para Etapa 2 da metodologia proposta. 
Os valores da fatura de energia são mostrados na Tabela 3 para cada Usina. Nessa tabela, podem se observar que as usinas com maiores potências instaladas e mais próximas das subestações dos sistemas de transmissão receberão valores maiores de remuneração. Em todos os casos do estudo aplicado se tem uma diminuição da fatura atual em valores próximos a 5 mil reais por mês.

Em trabalhos futuros, se analisará usinas com potências maiores a $5 \mathrm{MW}$ que sejam parte do mercado livre. Tal classe de usinas tem um contrato diferenciado de energia com penalizações nos casos que não cumpram com a injeção de potência ativa contrata. Igualmente, se quantificará por meio de fluxos de carga os benefícios que possam trazer para a operação da rede elétrica a injeção de potência reativa das usinas de açúcar e álcool. Tal análise permitirá quantificar melhor o valor taxa de remuneração, segundo as melhoras no perfil de tensão e diminuição das perdas elétricas. .

\begin{tabular}{|c|c|c|c|}
\hline $\begin{array}{c}\text { Nome da } \\
\text { Usina }\end{array}$ & $\begin{array}{c}\text { Fator de } \\
\text { Potência }\end{array}$ & $\begin{array}{c}\text { Valor de } \\
\text { Remuneração } \\
\text { TER } \\
\text { (R\$/Mvarh) }\end{array}$ & $\begin{array}{c}\text { Fatura de } \\
\text { Energia (R\$) }\end{array}$ \\
\hline Usina 1 & 0,954 & 9,2065 & 12.951 \\
\hline Usina 2 & 0,960 & 9,2005 & 22.776 \\
\hline Usina 3 & 0,954 & 9,1219 & 2.1931 \\
\hline Usina 4 & 0.945 & 9,1315 & 11.591 \\
\hline Usina 5 & 0.954 & 9,1997 & 19.536 \\
\hline Usina 6 & 0.945 & 9,1059 & 29.211 \\
\hline Usina 7 & 0.960 & 9,2005 & 26.941 \\
\hline
\end{tabular}

Tabela 3. Resultados da Etapa 2 da Metodologia proposta.

\section{CONCLUSÕES}

Neste trabalho se tem apresentado uma metodologia para analisar o mercado potencial de serviços ancilares de potência reativa quando providos pelas usinas de açúcar e álcool. Os resultados da metodologia proposta são a identificação das usinas que se encontram mais próximas das redes de transmissão e os valores de remuneração necessários para que os donos das usinas possam diminuir sua fatura de energia elétrica. Tal classe de resultados pode ajudar aos operadores do sistema elétrico na tomada de decisão, uma vez que facilita a operação do sistema elétrico de potência aproveitando recursos de potência reativa disponíveis em diversas partes da rede elétrica.

O mapa de saída da primeira etapa da proposta permite identificar fontes alternativas de reativos sem que haja necessidade de novos investimentos. Do ponto de vista econômico isto pode ajudar a diminuir o custo de investimento necessário para ter disponibilidade de serviços ancilares.
Os valores de remuneração calculados na segunda etapa permitem quantificar o incremento na remuneração que esta classe de usinas poderiam receber para que tenham uma diminuição na sua conta de energia elétrica. Assim, esta etapa auxilia aos planejadores na análise dos valores máximos e mínimos que possam ser aplicados de remuneração para estas fontes de injeção de reativos.

\section{AGRADECIMENTOS}

Os autores agradecem a INCT-INERGE e ao CNPq (Projeto Universal n. 432423/2016-8, 422044/2018-0) e à FAPESP (projeto 2017/22577-9, 2018/03015-2, 2019/00466-6) pelo auxílio financeiro.

\section{REFERÊNCIAS}

ANEEL - Agencia Nacional de Energia Elétrica Atualização da Resolução Normativa $n^{\circ} 414$, de 9 de setembro de 2010. Disponível em: http://www2.aneel.gov.br/cedoc/ren2010414comp.pdf. Acessado em 01 de Abril de 2019.

ANEEL - Agencia Nacional de Energia Elétrica - Resolução Homologatória $\mathrm{n}^{\circ} 2.498$, de 18 de dezembro de 2018. Disponível

http://www2.aneel.gov.br/cedoc/reh20182498ti.pdf. Acessado em 01 de Abril de 2019.

Brandao, R.; Oliveira, V. R.; Dorado, P.; Corrêa, P. V.; Carvalho, I. . Análise do mercado de serviços ancilares de. Portugal: Implicações para o caso brasileiro. In: 6th Latin American Energy Economics Meeting - New Energy Landscape: Impacts for Latin America, 2017, Rio de Janeiro. 6th Latin American Energy Economics Meeting - New Energy Landscape: Impacts for Latin America, 2017.

Cardoso de Lima, Gracieli Sartório ; Lopes, Elaine Coelho ; Motta, Juliana Gutierrez ; Asano, Roberto ; Valverde, María ; Suyama, Ricardo ; Leite, Patricia Teixeira . Sustainable Development Enhanced In The Decision Process Of Electricity Generation Expansion Planning. RENEWABLE ENERGY, V. 123, P. 563-577, 2018.

Cheng-Kang, Gao; Hong-Ming, Na; Kai-Hui, Song; Noel, Dyer; Fan, Tian, Qing-Jiang, Xu. Environmental impact analysis of power generation from biomass and wind farms in different locations. Renewable and Sustainable Energy Reviews 102 (2019) 307-317

EPE. WEBMAP EPE. Empresa de Pesquisa Energética, $2009 . \quad$ Disponivel em: http://www.epe.gov.br/Paginas/WEBMAP.aspx. Acessado em: 6 out. 2018.

GEOSEADE - PORTAL GEOSEADE. Fundação Seade.www.portalgeo.seade.gov.br/, Acesso em $11 \mathrm{fev}$ 2019. 
Hanashiro, D.; Belati, E. A., Utilização de Turbogeradores Instalados nas Usinas de Açúcar \& Etanol para Compensação de Reativos em Redes de Transmissão. In: INDUSCON- 2018, 2018, São Paulo. 13th IEEE/IAS International Conference on Industry Applications, 2018.

Khiaria, Besma; Jeguirimb, Mejdi. Biomass derived chars for energy applications. Renewable and Sustainable Energy Reviews 108 (2019) 253-273

KOTLER, Philip - Administração de Marketing - $10^{\mathrm{a}}$ Edição, $7^{\mathrm{a}}$ reimpressão - Tradução Bazán Tecnologia e Lingüística; revisão técnica Arão Sapiro. São Paulo: Prentice Hall, 2000, p.28.

Meehan, B. Modeling Electric Distribution with GIS. California: Esri Press, 2013.

ONS. Submódulo 14.1 - Administração dos serviços ancilares: visão geral Procedimentos de Rede, 2009. Disponível em: http://www.ons.org.br/\%2FProcedimentosDeRede\%2FM ódulo14\%2FSubmódulo4.1\%2FSubmódulo 14.1_Rev_1.0.pdf. Acessado em 01 de Abril de 2019.

Secretaria de Infraestrutura e Meio Ambiente do Estado de São Paulo. Zoneamento Agroambiental para o Setor Sucroalcooleiro. Disponível em: https://www.infraestruturameioambiente.sp.gov.br/etanol verde/zoneamento-agroambiental/. Acessado em Março de 2019.

Silva de Souza, Jeane; Molina, Yuri Percy; Silva de Araujo, Clivaldo; Pereira de Farias, Wendell; Santos de Araujo, Luri. Modified Particle Swarm Optimization Algorithm for Sizing photovoltaic System. IEEE Latin America Transsactions. Vol. 15, pp. 283-289, 2017.

UNICA - União da Indústria de Cana-de-Açúcar Bioeletricidade - Selo Energia Verde: Mais De 50 Usinas Certificadas Pelo Programa De Bioeletricidade, 2016. Disponível em: http://www.unica.com.br/noticia/1488364792032210178 2/selo-energia-verde-por-cento3A-mais-de-50-usinascertificadas-pelo-programa-de-bioeletricidade/.

Villavicencio, J.; Melo, J. D. and Padilha-Feltrin, A., "Hierarchical Bayesian Model For Estimating SpatialTemporal Photovoltaic Potential In Residential Areas," IEEE Trans. On Sustainable Energy, vol. 9, No. 2, pp. 971-979, May 2001. 